\title{
Equitable associate fuzzy graph of a fuzzy graph
}

\author{
M. Rani ${ }^{a}$, K. M. Dharmalingam ${ }^{b}$ \\ ${ }^{a}$ Department of Mathematics, Madurai Kamaraj University College Aundipatti,Tamilnadu State, India. \\ ${ }^{b}$ Department of Mathematics, The Madura College Madurai, Tamilnadu State, India.
}

\begin{abstract}
Let $\mathrm{G}=(\mathrm{V}, \sigma, \mu)$ be a fuzzy graph. Let $\mathrm{H}$ be the graph constructed from $\mathrm{G}$ as follows $\mathrm{V}(\mathrm{H})=\mathrm{V}(\mathrm{G})$, two points $\mathrm{u}$ and $\mathrm{v}$ are adjacent in $\mathrm{H}$ if and only if $\mathrm{u}$ and $\mathrm{v}$ are adjacent and degree fuzzy equitable in $\mathrm{G}$. $\mathrm{H}$ is called the adjacency inherent fuzzy equitable graph of $\mathrm{G}$ or fuzzy equitable associate graph of $G$ and is denoted by $e^{e f}(G)$. In this paper we introduced the concept of fuzzy equitable associate graph and obtain some interesting results for this new parameter in fuzzy equitable associate graph. (C)2016 All rights reserved.
\end{abstract}

Keywords: Fuzzy equitable dominating set, fuzzy equitable associate graph, pre-e-fuzzy equitable graph, degree equitable fuzzy graph.

2010 MSC: 05C72.

\section{Introduction}

L. A. Zadeh (1965) introduced the concepts of a fuzzy subset of a set as a way for representing uncertainty. His idea have been applied to a wide range of scientific areas.

Fuzzy concepts is also introduced in Graph theory. Formally, a fuzzy graph $G=(V, \sigma, \mu)$ is a non empty set $V$ together with a pair of functions $\sigma: V[0,1]$ and $\mu: V \times V[0,1]$ such that $\mu(u v) \leq \sigma(u) \wedge \sigma(v)$ for all $u, v$ in $V, \sigma$ is called the fuzzy vertex set of $G$ and $\mu$ is called the fuzzy edge set of $G$. The concept of equitable domination [10] in graphs was introduced by Venkatrasubramanian Swaminathan and Kuppusamy Markandan Dharmalingam. The notation of domination in fuzzy graphs [9] was developed by A. Somasundaram and S. Somasundaram. In this paper we introduced the concept of fuzzy equitable associate graph and obtain some interesting results for this new parameter in fuzzy equitable associate graph.

Email addresses: raniarul29@yahoo.com (M. Rani), kmdharma6902@yahoo.com (K. M. Dharmalingam) 


\section{Preliminaries}

Definition 2.1. A fuzzy graph $G=(\sigma, \mu)$ is a set with two functions $\sigma: \mathrm{V}[0,1]$ and $\mu: V \times V[0,1]$ such that $\mu(u v) \leq \sigma(u) \wedge \sigma(v)$ for all $u, v \in V$.

Definition 2.2. Let $G=(\sigma, \mu)$ be a fuzzy graph on $V$ and $V_{1} \subseteq V$. Define $\sigma_{1}$ on $V_{1}$ by $\sigma_{1}(u)=\sigma(u)$ for all $u \in V_{1}$ and $\mu_{1}$ on the collection $E_{1}$ of two element subset of $V_{1}$ by $\mu_{1}(u, v)=\mu(u, v)$ for all $u, v \in V_{1}$. Then $\left(\sigma_{1}, \mu_{1}\right)$ is called the fuzzy subgraph of $G$ induced by $V_{1}$ and is denoted by $\left\langle V_{1}\right\rangle$.

Definition 2.3. The degree of vertex $u$ is defined as the sum of the weights of the edges incident at $u$ and is denoted by $\operatorname{deg}(u)$.

Definition 2.4. Let $G$ be a fuzzy graph. Let $u$ and $v$ be two vertices of $G$. A subset $D$ of $V$ is called a fuzzy equitable dominating set if for every $v \in V-D$ there exist a vertex $u \in D$ such that $u v \in E(G)$ and $|\operatorname{deg}(u)-\operatorname{deg}(v)| \leq 1$ and $\mu(u v) \leq \sigma(u) \wedge \sigma(v)$. The minimum cardinality of a fuzzy equitable dominating set is denoted by $\gamma^{e f}$.

Definition 2.5. A vertex $u \in V$ is said to be degree equitable fuzzy graph with a vertex $v \in V$ if $|\operatorname{deg}(u)-\operatorname{deg}(v)| \leq 1$ and $\mu(u v) \leq \sigma(u) \wedge \sigma(v)$.

Definition 2.6. If $D$ is an fuzzy equitable dominating set then any super set of $D$ is a fuzzy equitable dominating set.

\section{Fuzzy Equitable Associate Graph}

Definition 3.1. Let $G=(V, \sigma, \mu)$ be a fuzzy graph. Let $H$ be the graph constructed from $\mathrm{G}$ as follows $V(H)=V(G)$, two points $\mathrm{u}$ and $\mathrm{v}$ are adjacent in $\mathrm{H}$ if and only if $\mathrm{u}$ and $\mathrm{v}$ are adjacent and degree fuzzy equitable in $G$. $H$ is called the fuzzy equitable associate graph $G$ and is denoted by $e^{e f}(G)$.

Remark 3.2.

(i) $e=u v \in E\left(e^{e f}(G)\right)$. Then $u$ and $v$ are adjacent and degree fuzzy equitable in $G$. Therefore $e^{e f} \in E(G), E\left(e^{e f}(G)\right) \subseteq E(G)$.

(ii) An edge $e=u v \in E(G)$ is said to be fuzzy equitable if $|\operatorname{deg}(v)-\operatorname{deg}(u)| \leq 1$ and $\mu(u v) \leq$ $\sigma(u) \wedge \sigma(v)$. Let $E^{e f}(G)$ be the set of all fuzzy equitable edges of $G$. Then clearly $E\left(e^{e f}(G)\right)=E^{e f}(G)$.

Remark 3.3. : $\overline{e^{e f}(G)}$ is need not be equal to $e^{e f}(\bar{G})$.

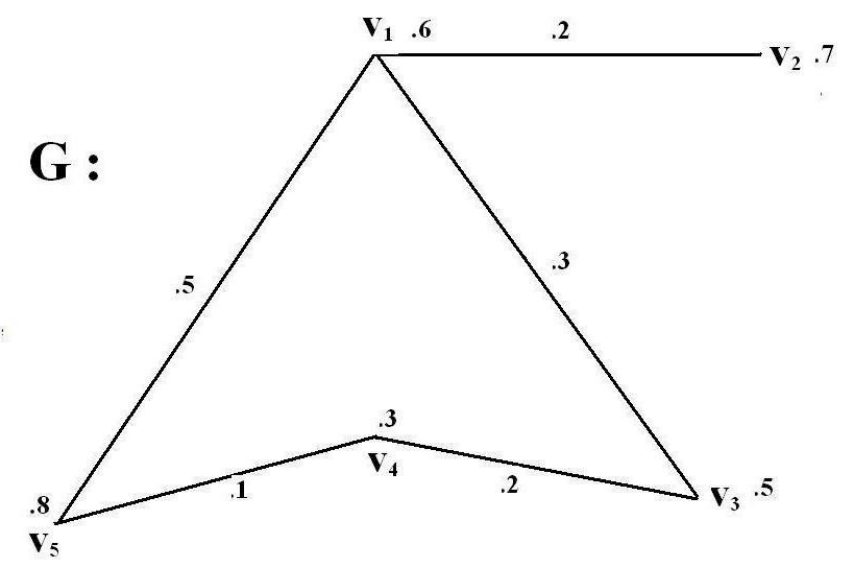



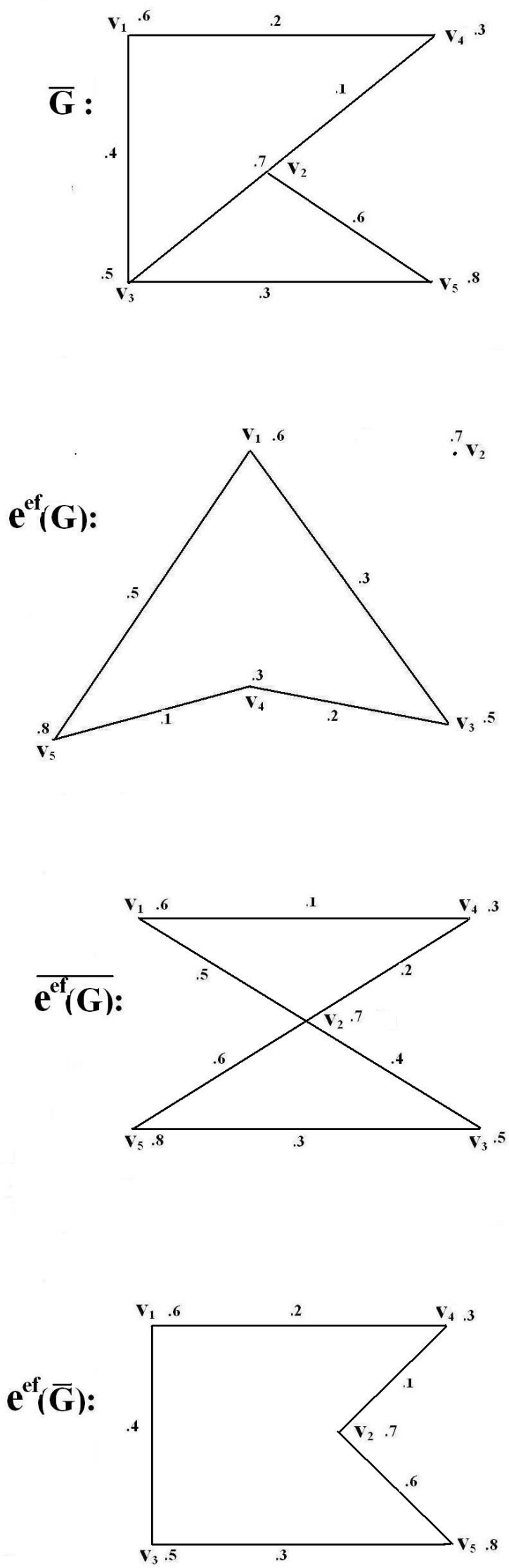
Therefore $\overline{e^{e f}(G)} \neq e^{e f}(\bar{G})$.

Theorem 3.4. $\overline{e^{e f}(G)}=e^{e f}(\bar{G})$ if and only if every edge of $G$ is fuzzy equitable.

Proof. Let every edge of $G$ is fuzzy equitable. Then $G=e^{e f}(G)$. Therefore $\bar{G}=\overline{e^{e f}(G)}$. Further, since every edge of $G$ is fuzzy equitable every edge of $\bar{G}$ is fuzzy equitable . Therefore $e^{e f}(\bar{G})=\bar{G}$. Therefore $e^{e f}(\bar{G})=\overline{e^{e f}(G)}$.

conversely, let $e^{e f}(\bar{G})=\overline{e^{e f}(G)}$. Suppose $G$ has a non-fuzzy equitable edge say $e=u v$. Then $u$ and $v$ are not adjacent in $e^{e f}(G)$ and hence $u$ and $v$ are adjacent in $\overline{e^{e f}(G)}$. Since $e=u v$ in $G, u$ and $v$ are not adjacent in $\bar{G}$ and hence $u$ and $v$ are not adjacent in $e^{e f}(\bar{G})$. Therefore $\overline{e^{e f}(G)} \neq e^{e f}(\bar{G})$ a contradiction. Hence every edge of $G$ is fuzzy equitable.

Definition 3.5. Let $G$ be a fuzzy graph and let $H$ be a fuzzy graph such that $e^{e f}(H)=G$. Then $H$ is called a pre-e-fuzzy equitable graph of $G$. Pre-e-fuzzy equitable graph of $G$ is not unique. The set of all pre-e-fuzzy equitable graph of $G$ is denoted by $\bar{e}^{e f}(G)$.
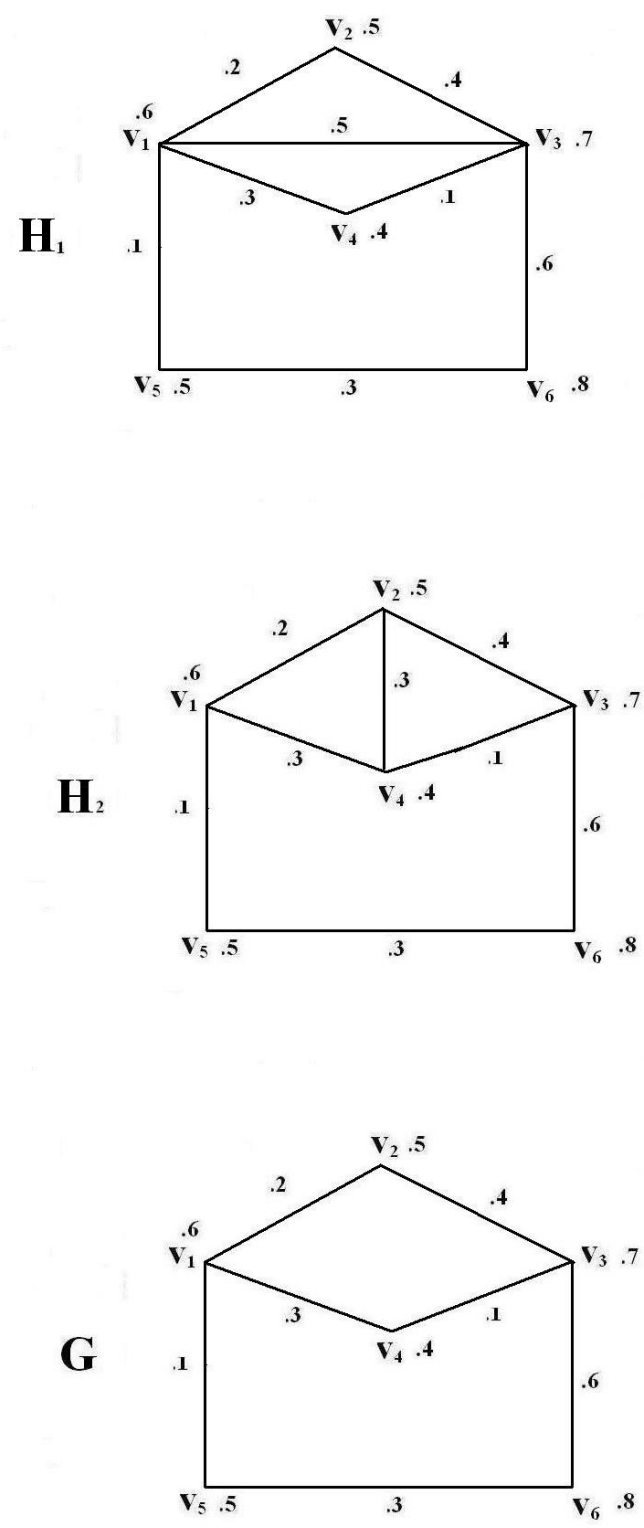
Therefore $H_{1}, H_{2} \in \bar{e}^{e f}(G)$.

Theorem 3.6. Let $G$ be a fuzzy graph. Let $H=e^{e f}(G)$. Then every edge of $H$ is degree equitable fuzzy graph if and only if the following holds: If $u$ and $v$ are degree fuzzy equitable and adjacent in $G$ then $d_{G}^{e f}(u)$ and $d_{G}^{e f}(v)$ are fuzzy equitable.

Proof. Let $H=e^{e f}(G)$. Therefore $E^{e f}(G)=E(H)$. Assume that if $u$ and $v$ are degree fuzzy equitable and adjacent in $G$, then $d_{G}^{e f}(u)$ and $d_{G}^{\text {ef }}(v)$ are fuzzy equitable. Let $e=u v \in E(H)$. Then $u$ and $v$ are degree fuzzy equitable in $G$. As $d_{H}^{e f}(u)=d_{G}^{e f}(u)$ and $d_{H}^{e f}(v)=d_{G}^{e f}(v)$ and as $d_{G}^{e f}(u)$ and $d_{G}^{e f}(v)$ are fuzzy equitable, we get that $u$ and $v$ are degree fuzzy equitable in $H$.

Conversely, assume that every edge of $\mathrm{H}$ is degree equitable. Let $e=u v \in E(H)$. Then $u$ and $v$ are degree fuzzy equitable and adjacent in $G$. Hence the theorem.

Theorem 3.7. If $G$ and $H$ are two fuzzy graphs, then $e^{e f}(G \square H)=e^{e f}(G) \square e^{e f}(H)$.

Proof. Let $(u, v),(x, y) \in V(G \square H)$ such that

$$
\begin{aligned}
& \left|d_{G \square H}^{e f}(u, v)-d_{G \square H}^{e f}(x, y)\right| \leq 1 \\
& \Rightarrow\left|d_{G}^{e f}(u)+d_{H}^{e f}(v)-d_{G}^{e f}(x)-d_{H}^{e f}(y)\right| \leq 1 \\
& \Rightarrow\left|\left[d_{G}^{e f}(u)-d_{G}^{e f}(x)\right]-\left[d_{H}^{e f}(v)-d_{H}^{e f}(y)\right]\right| \leq 1 \\
& \Rightarrow|| d_{G}^{e f}(u)-d_{G}^{e f}(x)|-| d_{H}^{e f}(v)-d_{H}^{e f}(y)|| \leq 1 .
\end{aligned}
$$

If $(u, v)$ and $(x, y)$ are adjacent in $e^{e f}(G \square H)$, then $(u, v)$ and $(x, y)$ are adjacent and degree fuzzy equitable in $G \square H$, either $u=x$ in $V(G)$ and $v y \in E(H)$ or $u x \in E(G)$ and $v=y$ in $V(H)$. Using (3.1) we get that either $u=x$ in $V(G)$ and $v$ and $y$ are adjacent and fuzzy equitable in $G$ and $v=y$ in $H$ or $u$ and $x$ are adjacent and fuzzy equitable in $G$ and $v=y$ in $V(H)$. i.e either $u=x$ in $V\left(e^{e f}(G)\right)$ and $u y \in E\left(e^{e f}(H)\right)$ or $u x \in E\left(e^{e f}(G)\right)$ and $v=y$ in $V\left(e^{e f}(H)\right)$. i.e $v=y$ in $V\left(e^{e f}(H)\right)$. i.e $(u, v)$ and $(x, y)$ are adjacent in $e^{e f}(G) \square e^{e f}(H)$.

Conversely, let $(u, v)$ and $(x, y)$ be adjacnet in $e^{e f}(G) \square e^{e f}(H)$. Then $u=x$ in $V\left(e^{e f}(G)\right)$ and $v y \in E\left(e^{e f}(H)\right)$ or $u x \in E\left(e^{e f}(G)\right)$ and $v=y$ in $V\left(e^{e f}(H)\right)$. i.e $u=x$ in $V(G)$ and $v$ and $y$ are adjacent and fuzzy equitable in $H$ or $u$ and $x$ are adjacent and fuzzy equitable in $G$ or $v=y$ in $V(H)$. This implies that $(u, v)$ and $(x, y)$ are adjacent in $(G \square H)$ and $\left|d_{G \square H}^{e f}(u, v)-d_{G \square H}^{e f}(x, y)\right|=$ $\left\|d_{G}^{e f}(u)-d_{G}^{e f}(x)|-| d_{H}^{e f}(y)-d_{H}^{e f}(v)\right\| \leq 1$. Since $u$ and $x$ are fuzzy equitable in $H$. Hence $(u, v),(x, y)$ are adjacent and degree fuzzy equitable in $G \square H$. That is $(\mathrm{u}, \mathrm{v})(\mathrm{x}, \mathrm{y})$ are adjacent in $e^{e f}(G \square H)$.

Theorem 3.8. For fuzzy graph $G$ such that $G$ and $\bar{G}$ have no fuzzy equitable isolates, then

$$
\gamma^{e f} e(G)+\gamma^{e f} e(\bar{G}) \leq n
$$

Proof. Since $G$ and $\bar{G}$ have no fuzzy equitable isolates $\gamma^{e f} e(G) \leq\left\lfloor\frac{n}{2}\right\rfloor \gamma^{e f} e(G) \leq\left\lfloor\frac{n}{2}\right\rfloor$. Therefore $\gamma^{e f} e(G)+\gamma^{e f} e(\bar{G}) \leq\left\lfloor\frac{n}{2}\right\rfloor+\left\lfloor\frac{n}{2}\right\rfloor \leq n$.

Theorem 3.9. Let $G$ be a fuzzy graph and every edges in $G$ is fuzzy equitable. Then

(i) $\gamma^{e f} e(G) \cdot \gamma^{e f} e(\bar{G}) \leq n$.

(ii) If $G$ and $\bar{G}$ have no isolates, then $\gamma^{e f} e(G)+\gamma^{e f} e(\bar{G}) \leq \frac{n}{2}+2$. 
Proof.

(i) Suppose $G$ is a fuzzy graph in which every edge is fuzzy equitable. Then $\gamma^{e f}(G)=\gamma^{e f} e(G)$. Also $\overline{e^{e f}(G)}=e^{e f}(\bar{G})$. Since every edge of $G$ is fuzzy equitable, every edge of $\bar{G}$ is fuzzy equitable and hence $\gamma^{e f}(\bar{G})=\gamma^{e f} e(\bar{G})$.

$\gamma^{e f} e(G) \cdot \overline{\gamma^{e f} e(G)} \leq n$ i.e $\gamma^{e f} e(G) \cdot \gamma^{e f} e(\bar{G}) \leq n$

(since $e(\bar{G})=\overline{e(G)}$ ) i.e $\gamma^{e f}(G) \cdot \gamma^{e f}(\bar{G}) \leq n$ Since $\gamma^{e f}(\bar{G})=\gamma^{e f} e(\bar{G})$.

(ii) Suppose $\mathrm{G}$ and have no isolates the $e^{e f}(G)$ and $e^{e f}(\bar{G})$ have no isolates, therefore $e(G)$ and $\overline{e(G)}$ have no isolates.

$\gamma^{e f} e(G)+\gamma^{e f} \overline{e(G)} \leq \frac{n}{2}+2$ i.e $\gamma^{e f} e(G)+\gamma^{e f} e(\bar{G}) \leq \frac{n}{2}+2$

i.e $\gamma^{e f}(G)+\gamma^{e f} \overline{(G)} \leq \frac{n}{2}+2$.

\section{Vizing's Conjecture for fuzzy Graphs}

A fuzzy graph $G=(\sigma, \mu)$ is said to satisfy Vizing's conjecture if $\gamma^{e f} e(G \square H) \gamma^{e f} e(G) \cdot \gamma^{e f} e(H)$ for every fuzzy graph $H$.

Theorem 4.1. Let $G=(\sigma, \mu)$ be a fuzzy graph satisfying Vizing's conjecture. Let $e=u v$ be an edge of $G$ such that $\mu(u v)=\sigma(u) \wedge \sigma(v)$ and $\gamma^{e f} e(G-e)=\gamma^{e f} e(G)$. Then $G-e$ also satisfying Vizing's conjecture.

Proof. The fuzzy graph $(G-e) \square H$ is a spanning subgraph of $G \square H$ for every fuzzy graph $H$. Hence $\gamma^{e f} e((G-e) \square H) \geq \gamma^{e f} e(G \square H) \geq \gamma^{e f} e(G) \cdot \gamma^{e f} e(H)=\gamma^{e f} e(G-e) \cdot \gamma^{e f} e(H)$. Thus $G-e$ also satisfies Vizing's conjecture.

\section{Conclusion}

There is scope for lot of research in the study of vulnerability of Networks. In addition to Interconnection networks where the study can be made, the mobile networks also provide an area for research. This paper we have introduced the concepts of fuzzy equtable domination and fuzzy equitable independent. Further study is being made on the total fuzzy equitable domination.

\section{References}

[1] E. J. Cockayne, S. T. Hedetniemi, Towards a theory of domination in graphs', Networks, (1977), 247261.

[2] K. M. Dharmalingam, M. Rani, Equitable Domination in Fuzzy Graphs', Int. J. Pure Appl. Math., 94 (2014), 661-667.

[3] Kuppusamy Markandan Dharmalingam, Equitable Associate Graph of a Graph, Bull. Int. Math. Virtual Inst., 2 (2012) 109-116.

[4] J. N Mordeson, P. S Nair, Fuzzy graphs and Fuzzy Hyper graphs", Physica Verlag, Heidelberg, (1998), Second edition (2001).

[5] A. Nagoor Gani, V. T. Chandra Sekaran, Domination in Fuzzy Graph, Adv. Fuzzy Sets Syst., 1 (2006), $17-26$.

[6] A. Nagoor Gani, P. Vadivel, Contribution to the theory of Domination, Independence and Irredundance in Fuzzy graph, Bull. Pure Appl. Sci. Sect. E Math. Stat., 28 (2009), 179-187.

[7] A. Rosenfield, Fuzzy graphs, in, L. A. Zadeh, K. S. Fu, M. Shimura (Eds), Fuzzy sets and their Applications to congnitive and Decision Processes, Academic Press, New York, (1975), 77-95. 
[8] E. Sampath Kumar, L. Puspalatha, Strong, weak domination and domination balance in a graph, Discrete Math., 161 (1966) 235-242.

[9] A. Somasundaram, S. Somasundaram, Domination in Fuzzy Graphs-I, Elsevier Science, 19 (1998), 787-791. 1

[10] V. Swaminathan, K. M. Dharmalingam, Degree Equitable Domination on Graphs, Kragujevac J. Math., 35 (2011), 191-197. 1

[11] Z. Tahmasbzadehbaee, N. D. Soner, D. A. Mojdeh, Neighborhood number in Graphs, J Math. Comput. Sci., 5 (2012), 265-270. 